\title{
Connection between interval-valued observables and intuitionistic fuzzy observables
}

\author{
Katarína Čunderlíková \\ Mathematical Institute, Slovak Academy of Sciences \\ 49 Stefánikova Str., 81473 Bratislava, Slovakia \\ e-mail: cunderlikova. lendelova@gmail.com
}

Received: 23 January 2019

Revised: 22 March 2019

Accepted: 28 March 2019

\begin{abstract}
In paper [4] the authors studied probability on two lattices and they showed that these two lattices are isomorphic. First lattice was the geometrical interpretation of intuitionistic fuzzy sets introduced by K. T. Atanassov and the second lattice was the geometrical interpretation of interval valued sets introduced by L. A. Zadeh. Later in papers [3, 6] authors studied intuitionistic fuzzy events and interval-valued events. They showed that these two systems are isomorphic and they illustrated the connection between intuitionistic fuzzy state and interval valued state. In this paper, we define the notion of interval valued observable and we display the connection to the intuitionistic fuzzy observable. We define the notion of interval valued mean value and dispersion and we show the relation between interval-valued distribution function and intuitionistic fuzzy distribution function, too.
\end{abstract}

Keywords: Intuitionistic fuzzy set, Interval-valued set, Intuitionistic fuzzy event, Interval-valued event, Intuitionistic fuzzy state, Interval-valued state, Intuitionistic fuzzy observable, Intervalvalued observable, Isomorphism, Interval-valued mean value, Interval-valued dispersion, Intervalvalued distribution function, Intuitionistic fuzzy distribution function.

2010 Mathematics Subject Classification: 03E72, 03B52, 60A86.

\section{Introduction}

In paper [4] K. Lendelová and A. Michalíková studied probability on two lattices $\left(L^{*}, \leq_{L^{*}}\right)$ and $\left(L^{1}, \leq_{L^{1}}\right)$. The first lattice $\left(L^{*}, \leq_{L^{*}}\right)$ given by 


$$
\begin{gathered}
L^{*}=\left\{(x, y) \mid(x, y) \in[0,1]^{2} \text { and } x+y \leq 1\right\} \\
\left(x_{1}, y_{1}\right) \leq L^{*}\left(x_{2}, y_{2}\right) \Leftrightarrow x_{1} \leq x_{2} \text { and } y_{1} \geq y_{2} \forall\left(x_{1}, y_{1}\right),\left(x_{2}, y_{2}\right) \in L^{*}
\end{gathered}
$$

(see Figure 1) is the geometrical interpretation of intuitionistic fuzzy sets introduced by K. T. Atanassov in $[1,2]$. They used for each $\left(x_{1}, y_{1}\right),\left(x_{2}, y_{2}\right) \in L^{*}$ the Łukasiewicz connectives $\oplus$, $\odot$ defined by

$$
\begin{aligned}
& \left(x_{1}, y_{1}\right) \oplus\left(x_{2}, y_{2}\right)=\left(\left(x_{1}+x_{2}\right) \wedge 1,\left(y_{1}+y_{2}-1\right) \vee 0\right) \\
& \left(x_{1}, y_{1}\right) \odot\left(x_{2}, y_{2}\right)=\left(\left(x_{1}+x_{2}-1\right) \vee 0,\left(y_{1}+y_{2}\right) \wedge 1\right)
\end{aligned}
$$

where $\wedge=\min$ and $\vee=\max$.

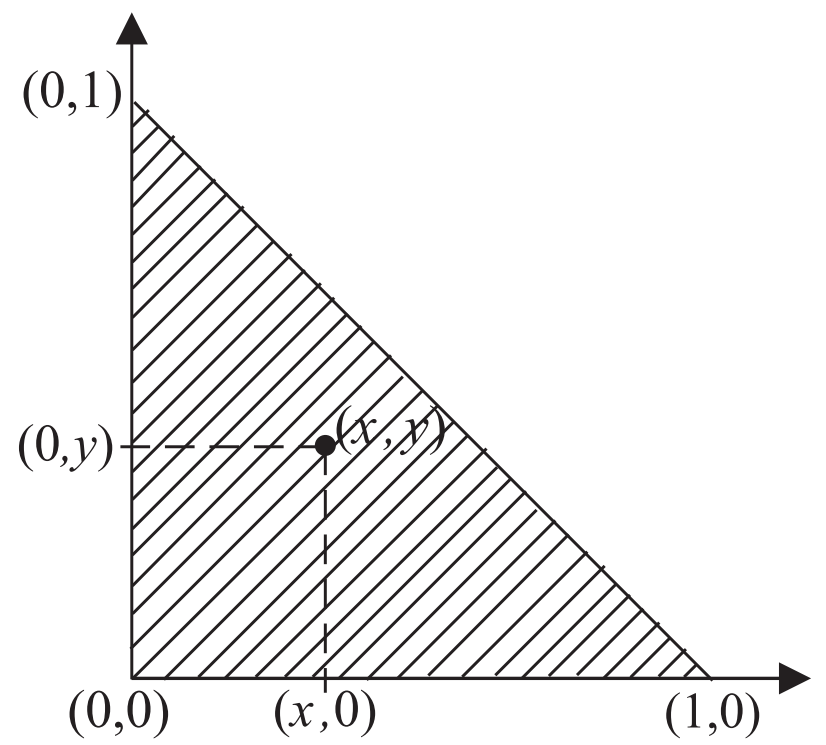

Figure 1. The shaded area constitutes the set $L^{*}$.

The second lattice $\left(L^{1}, \leq_{L^{1}}\right)$ given by

$$
\begin{gathered}
L^{1}=\left\{(x, y) \mid(x, y) \in[0,1]^{2} \text { and } x \leq y\right\} \\
\left(x_{1}, y_{1}\right) \leq_{L^{1}}\left(x_{2}, y_{2}\right) \Leftrightarrow x_{1} \leq x_{2} \text { and } y_{1} \leq y_{2} \forall\left(x_{1}, y_{1}\right),\left(x_{2}, y_{2}\right) \in L^{1}
\end{gathered}
$$

(see Figure 2) is the geometrical interpretation of interval-valued sets introduced by L. A. Zadeh in [10]. They used for $\left(x_{1}, y_{1}\right),\left(x_{2}, y_{2}\right) \in L^{1}$ the operations $\widehat{\oplus}$ and $\widehat{\odot}$ defined by

$$
\begin{aligned}
\left(x_{1}, y_{1}\right) \widehat{\oplus}\left(x_{2}, y_{2}\right) & =\left(\left(x_{1}+x_{2}\right) \wedge 1,\left(y_{1}+y_{2}\right) \wedge 1\right) \\
\left(x_{1}, y_{1}\right) \widehat{\odot}\left(x_{2}, y_{2}\right) & =\left(\left(x_{1}+x_{2}-1\right) \vee 0,\left(y_{1}+y_{2}-1\right) \vee 0\right)
\end{aligned}
$$

where $\wedge=\min$ and $\vee=\max$.

K. Lendelová and A. Michalíková showed that these two lattices with their operations are isomorphic by the one-to-one correspondence $\varphi: L^{1} \rightarrow L^{*}$ defined by

$$
\varphi((x, y))=(x, 1-y)
$$




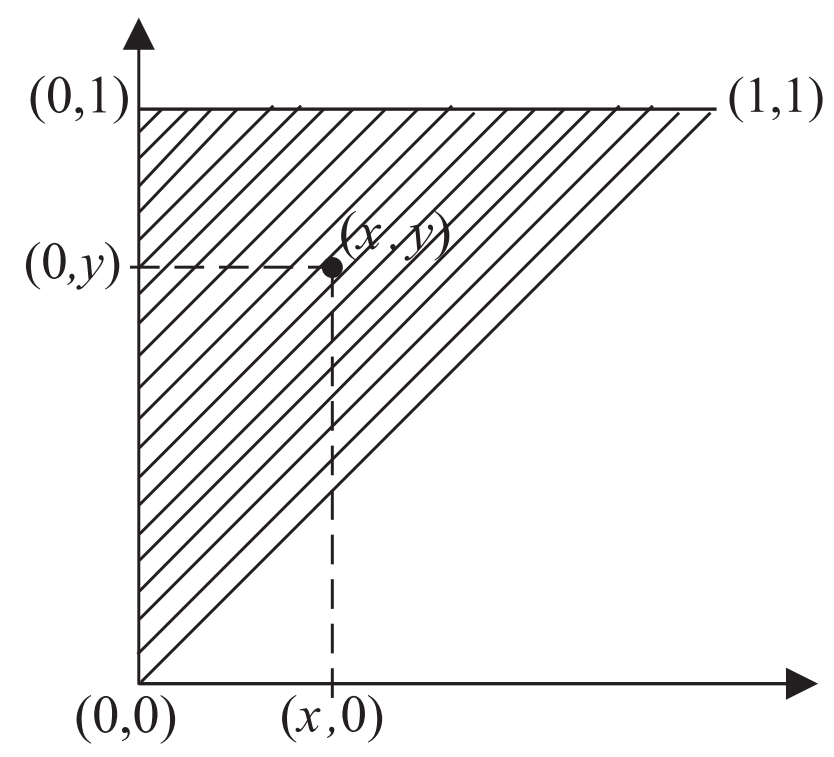

Figure 2. The shaded area constitutes the set $L^{1}$.

for each $(x, y) \in L^{1}$. Therefore, the following relations hold

$$
\begin{aligned}
\left(x_{1}, y_{1}\right) \leq_{L^{1}}\left(x_{2}, y_{2}\right) & \Leftrightarrow \varphi\left(\left(x_{1}, y_{1}\right)\right) \leq_{L^{*}} \varphi\left(\left(x_{2}, y_{2}\right)\right) \\
\varphi\left(\left(x_{1}, y_{1}\right) \widehat{\oplus}\left(x_{2}, y_{2}\right)\right) & =\varphi\left(\left(x_{1}, y_{1}\right)\right) \oplus \varphi\left(\left(x_{2}, y_{2}\right)\right) \\
\varphi\left(\left(x_{1}, y_{1}\right) \widehat{\odot}\left(x_{2}, y_{2}\right)\right) & =\varphi\left(\left(x_{1}, y_{1}\right)\right) \odot \varphi\left(\left(x_{2}, y_{2}\right)\right)
\end{aligned}
$$

for each $\left(x_{1}, y_{1}\right),\left(x_{2}, y_{2}\right) \in L^{1}$

Later in papers [3, 6] B. Riečan, P. Král and A. Michalíková studied a connection between the family of intuitionistic fuzzy events

$$
\mathcal{F}=\left\{\left(\mu_{A}, \nu_{A}\right) \mid \mu_{A}+\nu_{A} \leq 1_{\Omega} \text { and } \mu_{A}, \nu_{A}: \Omega \rightarrow[0,1] \text { are } \mathcal{S} \text {-measurable functions }\right\}
$$

with the operations and relation

$$
\begin{aligned}
& \mathbf{A} \leq \mathbf{B} \Leftrightarrow \mu_{A} \leq \mu_{B}, \nu_{A} \geq \nu_{B}, \\
& \mathbf{A} \oplus \mathbf{B}=\left(\left(\mu_{A}+\mu_{B}\right) \wedge 1_{\Omega},\left(\nu_{A}+\nu_{B}-1_{\Omega}\right) \vee 0_{\Omega}\right), \\
& \left.\mathbf{A} \odot \mathbf{B}=\left(\left(\mu_{A}+\mu_{B}-1_{\Omega}\right) \vee 0_{\Omega},\left(\nu_{A}+\nu_{B}\right) \wedge 1_{\Omega}\right)\right) .
\end{aligned}
$$

and the family of interval-valued events

$$
\mathcal{K}=\left\{\left(\pi_{C}, \rho_{C}\right) \mid \pi_{C} \leq \rho_{C} \text { and } \pi_{C}, \rho_{C}: \Omega \rightarrow[0,1] \text { are } \mathcal{S} \text {-measurable functions }\right\}
$$

with the operations and relation

$$
\begin{aligned}
\mathbf{C} \preceq \mathbf{D} & \Leftrightarrow \pi_{C} \leq \pi_{D}, \rho_{C} \leq \rho_{D} \\
\mathbf{C} \widehat{\oplus} \mathbf{D} & =\left(\left(\pi_{C}+\pi_{D}\right) \wedge 1_{\Omega},\left(\rho_{C}+\rho_{D}\right) \wedge 1_{\Omega}\right) \\
\mathbf{C} \widehat{\odot} \mathbf{D} & =\left(\left(\pi_{C}+\pi_{D}-1_{\Omega}\right) \vee 0_{\Omega},\left(\rho_{C}+\rho_{D}-1_{\Omega}\right) \vee 0_{\Omega}\right) .
\end{aligned}
$$


They showed that these two systems are isomorphic by the mapping $\psi: \mathcal{F} \rightarrow \mathcal{K}$ given by

$$
\psi\left(\left(\mu_{A}, \nu_{A}\right)\right)=\left(\mu_{A}, 1_{\Omega}-\nu_{A}\right)
$$

for each $\mathbf{A}=\left(\mu_{A}, \nu_{A}\right) \in \mathcal{F}$. Therefore, the following relations hold

$$
\begin{aligned}
\psi(\mathbf{A} \oplus \mathbf{B}) & =\psi(\mathbf{A}) \widehat{\oplus} \psi(\mathbf{B}), \\
\psi(\mathbf{A} \odot \mathbf{B}) & =\psi(\mathbf{A}) \widehat{\odot} \psi(\mathbf{B}), \\
\mathbf{A} \leq \mathbf{B} & \Leftrightarrow \psi(\mathbf{A}) \preceq \psi(\mathbf{B}), \\
\mathbf{A}_{n} \nearrow \mathbf{A} & \Leftrightarrow \psi\left(\mathbf{A}_{n}\right) \nearrow \psi(\mathbf{A}),
\end{aligned}
$$

for each $\mathbf{A}_{n}, \mathbf{A}, \mathbf{B} \in \mathcal{F}$. They illustrated the connection between intuitionistic fuzzy state $\mathbf{m}: \mathcal{F} \rightarrow[0,1]$ and interval-valued state $k: \mathcal{K} \rightarrow[0,1]$ and that was $\mathbf{m}=k \circ \psi$.

In this paper, we define the notion of interval-valued observable and we display the connection to the intuitionistic fuzzy observable, too. First the notion of intuitionistic fuzzy observable $x: \mathcal{B}(R) \rightarrow \mathcal{F}$ was introduced in paper [5]. We define the notion of interval-valued mean value and dispersion and we show the connection between the interval-valued distribution function and the intuitionistic fuzzy distribution function, too.

Remark that in a whole text we use a notation "IF" for short a phrase "intuitionistic fuzzy" and a notation "IV" for short a phrase "interval-valued".

\section{Interval-valued events and interval-valued states}

First we start with definitions of basic notions (see $[3,6])$.

Definition 2.1 Let $\Omega$ be a nonempty set. An interval-valued set (IV-set) $\mathrm{C}$ on $\Omega$ is a pair $\left(\pi_{C}, \rho_{C}\right)$ of mappings $\pi_{C}, \rho_{C}: \Omega \rightarrow[0,1]$ such that $\pi_{C} \leq \rho_{C}$.

Definition 2.2 Start with a measurable space $(\Omega, \mathcal{S})$. Hence $\mathcal{S}$ is a $\sigma$-algebra of subsets of $\Omega$. An interval-valued event (IV-event) is called an IV-set $\mathbf{C}=\left(\pi_{C}, \rho_{C}\right)$ such that $\pi_{C}, \rho_{C}: \Omega \rightarrow[0,1]$ are $\mathcal{S}$-measurable. The family of all IV-events on $(\Omega, \mathcal{S})$ will be denoted by $\mathcal{K}$.

If $\mathbf{C}=\left(\pi_{C}, \rho_{C}\right) \in \mathcal{K}, \mathbf{D}=\left(\pi_{D}, \rho_{D}\right) \in \mathcal{K}$, then we define the Łukasiewicz binary operations $\widehat{\oplus}, \widehat{\odot}$ on $\mathcal{K}$ by

$$
\begin{aligned}
& \mathbf{C} \widehat{\oplus} \mathbf{D}=\left(\left(\pi_{C}+\pi_{D}\right) \wedge 1_{\Omega},\left(\rho_{C}+\rho_{D}\right) \wedge 1_{\Omega}\right) \\
& \mathbf{C} \widehat{\odot} \mathbf{D}=\left(\left(\pi_{C}+\pi_{D}-1_{\Omega}\right) \vee 0_{\Omega},\left(\rho_{C}+\rho_{D}-1_{\Omega}\right) \vee 0_{\Omega}\right)
\end{aligned}
$$

and the partial ordering is given by

$$
\mathbf{C} \preceq \mathbf{D} \Leftrightarrow \pi_{C} \leq \pi_{D}, \rho_{C} \leq \rho_{D}
$$


The continuity is given by

$$
\begin{aligned}
& \mathbf{C} \nearrow \mathbf{D} \Leftrightarrow \pi_{C} \nearrow \pi_{D}, \rho_{C} \nearrow \rho_{D}, \\
& \mathbf{C} \searrow \mathbf{D} \Leftrightarrow \pi_{C} \searrow \pi_{D}, \rho_{C} \searrow \rho_{D} .
\end{aligned}
$$

In the IV-probability theory instead of the notion of probability we use the notion of state (see $[3,6])$.

Definition 2.3 Let $\mathcal{K}$ be the family of all IV-events in $\Omega$. A mapping $k: \mathcal{K} \rightarrow[0,1]$ is called an interval valued state (IV-state), if the following conditions are satisfied:

(i) $k\left(\left(1_{\Omega}, 0_{\Omega}\right)\right)=1, k\left(\left(0_{\Omega}, 0_{\Omega}\right)\right)=0$;

(ii) if $\mathbf{C} \widehat{\odot} \mathbf{D}=\left(0_{\Omega}, 0_{\Omega}\right)$ and $\mathbf{C}, \mathbf{D} \in \mathcal{K}$, then $k(\mathbf{C} \widehat{\oplus} \mathbf{D})=k(\mathbf{C})+k(\mathbf{D})$;

(iii) if $\mathbf{C}_{n} \nearrow \mathbf{C}$ (i.e. $\left.\pi_{C_{n}} \nearrow \pi_{C}, \rho_{C_{n}} \nearrow \rho_{C}\right)$, then $k\left(\mathbf{C}_{n}\right) \nearrow k(\mathbf{C})$.

Probably the most useful result in the IV-state theory is the following representation theorem.

Theorem 2.4 To each IV-state $k: \mathcal{K} \rightarrow[0,1]$ there exists exactly one probability measure $P: \mathcal{S} \rightarrow[0,1]$ and exactly one $\alpha \in[0,1]$ such that

$$
k(\mathbf{C})=(1-\alpha) \int_{\Omega} \pi_{C} d P+\alpha \int_{\Omega} \rho_{C} d P
$$

for each $\mathbf{C}=\left(\pi_{C}, \rho_{C}\right) \in \mathcal{K}$.

Between IV-states and IF-states is one-to-one correspondence by the mapping $\psi: \mathcal{F} \rightarrow \mathcal{K}$ given by

$$
\psi\left(\left(\mu_{A}, \nu_{A}\right)\right)=\left(\mu_{A}, 1_{\Omega}-\nu_{A}\right)
$$

for each $\mathbf{A}=\left(\mu_{A}, \nu_{A}\right) \in \mathcal{F}$. About this says the following proposition.

Proposition 2.1 If $k: \mathcal{K} \rightarrow[0,1]$ is an IV-state and $\mathbf{m}=k \circ \psi: \mathcal{F} \rightarrow[0,1]$, then $\mathbf{m}$ is an IF-state.

Recall that by an intuitionistic fuzzy state (IF-state) $\mathbf{m}$ we understand each mapping $\mathbf{m}: \mathcal{F} \rightarrow[0,1]$ which satisfies the following conditions (see [7]):

(i) $\mathbf{m}\left(\left(1_{\Omega}, 0_{\Omega}\right)\right)=1, \mathbf{m}\left(\left(0_{\Omega}, 1_{\Omega}\right)\right)=0$;

(ii) if $\mathbf{A} \odot \mathbf{B}=\left(0_{\Omega}, 1_{\Omega}\right)$ and $\mathbf{A}, \mathbf{B} \in \mathcal{F}$, then $\mathbf{m}(\mathbf{A} \oplus \mathbf{B})=\mathbf{m}(\mathbf{A})+\mathbf{m}(\mathbf{B})$;

(iii) if $\mathbf{A}_{n} \nearrow \mathbf{A}$ (i.e. $\left.\mu_{A_{n}} \nearrow \mu_{A}, \nu_{A_{n}} \searrow \nu_{A}\right)$, then $\mathbf{m}\left(\mathbf{A}_{n}\right) \nearrow \mathbf{m}(\mathbf{A})$. 


\section{Interval-valued observables}

The third basic notion in the probability theory is the notion of an observable. Let $\mathcal{J}$ be the family of all intervals in $R$ of the form

$$
[a, b)=\{x \in R: a \leq x<b\} .
$$

Then the $\sigma$-algebra $\sigma(\mathcal{J})$ is denoted $\mathcal{B}(R)$ and it is called the $\sigma$-algebra of Borel sets, its elements are called Borel sets.

Definition 3.1 By an interval-valued observable (IV-observable) on $\mathcal{K}$ we understand each mapping $z: \mathcal{B}(R) \rightarrow \mathcal{K}$ satisfying the following conditions:

(i) $z(R)=\left(1_{\Omega}, 1_{\Omega}\right), z(\emptyset)=\left(0_{\Omega}, 0_{\Omega}\right)$;

(ii) if $A \cap B=\emptyset$, then $z(A) \widehat{\odot} z(B)=\left(0_{\Omega}, 0_{\Omega}\right)$ and $z(A \cup B)=z(A) \widehat{\oplus} z(B)$;

(iii) if $A_{n} \nearrow A$, then $z\left(A_{n}\right) \nearrow z(A)$.

Theorem 3.2 Let $z: \mathcal{B}(R) \rightarrow \mathcal{K}$ be an IV-observable on $\mathcal{K}$. If we denote $z(A)=\left(z^{b}(A), z^{\sharp}(A)\right)$ for each $A \in \mathcal{B}(R)$, then $z^{b}, z^{\sharp}: \mathcal{B}(R) \rightarrow \mathcal{T}$ are observables, where $\mathcal{T}=\{f: \Omega \rightarrow$ $[0,1] ; f$ is $\mathcal{S}$-measurable $\}$.

Proof. Since $\left(1_{\Omega}, 1_{\Omega}\right)=z(R)=\left(z^{b}(R), z^{\sharp}(R)\right),\left(0_{\Omega}, 0_{\Omega}\right)=z(\emptyset)=\left(z^{b}(\emptyset), z^{\sharp}(\emptyset)\right)$, then

$$
\begin{aligned}
& z^{b}(R)=1_{\Omega}, z^{b}(\emptyset)=0_{\Omega}, \\
& z^{\sharp}(R)=1_{\Omega}, z^{\sharp}(\emptyset)=0_{\Omega} .
\end{aligned}
$$

Let $A \cap B=\emptyset$, then

$$
\begin{aligned}
\left(0_{\Omega}, 0_{\Omega}\right) & =z(A) \widehat{\odot} z(B)=\left(z^{b}(A), z^{\sharp}(A)\right) \widehat{\odot}\left(z^{b}(B), z^{\sharp}(B)\right) \\
& =\left(z^{b}(A) \odot z^{b}(B), z^{\sharp}(A) \odot z^{\sharp}(B)\right) .
\end{aligned}
$$

Hence $z^{b}(A) \odot z^{b}(B)=0_{\Omega}, z^{\sharp}(A) \odot z^{\sharp}(B)=0_{\Omega}$. Moreover

$$
\begin{aligned}
\left(z^{b}(A \cup B), z^{\sharp}(A \cup B)\right) & =z(A \cup B)=z(A) \widehat{\oplus} z(B) \\
& =\left(z^{b}(A) \oplus z^{b}(B), z^{\sharp}(A) \oplus z^{\sharp}(B)\right),
\end{aligned}
$$

hence

$$
\begin{aligned}
& z^{b}(A \cup B)=z^{b}(A) \oplus z^{b}(B), \\
& z^{\sharp}(A \cup B)=z^{\sharp}(A) \oplus z^{\sharp}(B) .
\end{aligned}
$$

Finally, let $A_{n} \nearrow A$. Then

$$
\left(z^{b}\left(A_{n}\right), z^{\sharp}\left(A_{n}\right)\right)=z\left(A_{n}\right) \nearrow z(A)=\left(z^{b}(A), z^{\sharp}(A)\right),
$$

therefore,

$$
z^{b}\left(A_{n}\right) \nearrow z^{b}(A), z^{\sharp}\left(A_{n}\right) \nearrow z^{\sharp}(A) .
$$

This completes the proof. 
Remark 3.3 Sometimes we need to work with an n-dimensional IV-observable $z: \mathcal{B}\left(R^{n}\right) \rightarrow \mathcal{K}$ defined as a mapping with the following conditions:

(i) $z\left(R^{n}\right)=\left(1_{\Omega}, 1_{\Omega}\right), z(\emptyset)=\left(0_{\Omega}, 0_{\Omega}\right)$;

(ii) if $A \cap B=\emptyset, A, B \in \mathcal{B}\left(R^{n}\right)$, then $z(A) \widehat{\odot} z(B)=\left(0_{\Omega}, 0_{\Omega}\right)$ and $z(A \cup B)=z(A) \widehat{\oplus} z(B)$;

(iii) if $A_{n} \nearrow A$, then $z\left(A_{n}\right) \nearrow z(A)$ for each $A, A_{n} \in \mathcal{B}\left(R^{n}\right)$.

If $n=1$ we simply say that $z$ is an IV-observable.

Now we show a connection between an IV-observable and an IF-observable. Recall that by intuitionistic fuzzy observable (IF-observable) on $\mathcal{F}$ we understand each mapping $x: \mathcal{B}(R) \rightarrow$ $\mathcal{F}$ satisfying the following conditions (see $[5,8])$ :

(i) $x(R)=\left(1_{\Omega}, 0_{\Omega}\right), x(\emptyset)=\left(0_{\Omega}, 1_{\Omega}\right)$;

(ii) if $A \cap B=\emptyset$, then $x(A) \odot x(B)=\left(0_{\Omega}, 1_{\Omega}\right)$ and $x(A \cup B)=x(A) \oplus x(B)$;

(iii) if $A_{n} \nearrow A$, then $x\left(A_{n}\right) \nearrow x(A)$.

If we denote $x(A)=\left(x^{b}(A), 1-x^{\sharp}(A)\right)$ for each $A \in \mathcal{B}(R)$, then $x^{b}, x^{\sharp}: \mathcal{B}(R) \rightarrow \mathcal{T}$ are observables, where $\mathcal{T}=\{f: \Omega \rightarrow[0,1] ; f$ is $\mathcal{S}$-measurable $\}$.

Proposition 3.1 Let $\psi: \mathcal{F} \rightarrow \mathcal{K}, \psi((u, v))=\left(u, 1_{\Omega}-v\right)$. If $x: \mathcal{B}(R) \rightarrow \mathcal{F}$ is an IF-observable and $z=\psi \circ x: \mathcal{B}(R) \rightarrow \mathcal{K}$, then $z$ is an IV-observable.

Proof. Let $x: \mathcal{B}(R) \rightarrow \mathcal{F}$ be an IF-observable, $\psi((u, v))=\left(u, 1_{\Omega}-v\right)$. Put $z=\psi \circ x$. Then

$$
\begin{aligned}
z(R) & =\psi(x(R))=\psi\left(\left(1_{\Omega}, 0_{\Omega}\right)\right)=\left(1_{\Omega}, 1_{\Omega}-0_{\Omega}\right)=\left(1_{\Omega}, 1_{\Omega}\right), \\
z(\emptyset) & =\psi(x(\emptyset))=\psi\left(\left(0_{\Omega}, 1_{\Omega}\right)\right)=\left(0_{\Omega}, 1_{\Omega}-1_{\Omega}\right)=\left(0_{\Omega}, 0_{\Omega}\right) .
\end{aligned}
$$

Let $A, B \in R$. If $A \cap B=\emptyset$, then $x(A) \odot x(B)=\left(0_{\Omega}, 1_{\Omega}\right)$ and using (2) we have

$$
\begin{aligned}
z(A) \widehat{\odot} z(B) & =\psi(x(A)) \widehat{\odot} \psi(x(B))=\psi(x(A) \odot x(B)) \\
& =\psi\left(\left(0_{\Omega}, 1_{\Omega}\right)\right)=\left(0_{\Omega}, 0_{\Omega}\right) .
\end{aligned}
$$

Moreover, using (1), we obtain

$$
\begin{aligned}
z(A \cup B) & =\psi(x(A \cup B))=\psi(x(A) \oplus x(B))=\psi(x(A)) \widehat{\oplus} \psi(x(B)) \\
& =z(A) \widehat{\oplus} z(B) .
\end{aligned}
$$

Finally, let $A_{n} \nearrow A$. Then $x\left(A_{n}\right) \nearrow x(A)$ and by (4)

$$
z\left(A_{n}\right)=\psi\left(x\left(A_{n}\right)\right) \nearrow \psi(x(A))=z(A) .
$$

Therefore, $z$ is an IV-observable. 


\section{Interval-valued mean value and dispersion}

In this section, we define the notion of interval-valued mean value and dispersion for IV-observable. Similarly as in the classical case the following theorem can be proved.

Theorem 4.1 Let $z: \mathcal{B}(R) \rightarrow \mathcal{K}$ be an IV-observable, $k: \mathcal{K} \rightarrow[0,1]$ be an IV-state. Define the mapping $k_{z}: \mathcal{B}(R) \rightarrow[0,1]$ by the formula

$$
k_{z}(C)=k(z(C))
$$

for each $C \in \mathcal{B}(R)$. Then $k_{z}: \mathcal{B}(R) \rightarrow[0,1]$ is a probability measure. Moreover,

$$
k_{z}(C)=\mathbf{m}_{x}(C)
$$

where $\mathbf{m}_{x}=\mathbf{m} \circ x$ is a probability measure induced by IF-state $\mathbf{m}$ and IF-observable $x$.

Proof. Let $z: \mathcal{B}(R) \rightarrow \mathcal{K}$ be an $I V$-observable, $k: \mathcal{K} \rightarrow[0,1]$ be an IV-state. Put $\psi((u, v))=$ $\left(u, 1_{\Omega}-v\right)$.

Using Proposition 3.1, there exists an IF-observable $x=\psi^{-1} \circ z$ such that $z=\psi \circ x$ and by Proposition 2.1 there exists an IF-state $\mathbf{m}$ such that $\mathbf{m}=k \circ \psi$. Hence

$$
k_{z}(C)=k(z(C))=k(\psi \circ x(C))=\mathbf{m}(x(C))=\mathbf{m}_{x}(C)
$$

for each $C \in \mathcal{B}(R)$.

Remark 4.2 Theorem 4.1 says that $k_{z}$ and $\mathbf{m}_{x}$ are the same probability measures.

Recall now the Kolmogorov case. If $\xi: \Omega \rightarrow R$ is a random variable, then

$$
E(\xi)=\int_{\Omega} \xi d P=\int_{R} t d P_{\xi}(t)
$$

where

$$
P_{\xi}(B)=P\left(\xi^{-1}(B)\right) .
$$

Since now $k_{z}: \mathcal{B}(R) \rightarrow[0,1]$ plays an analogous role as $P_{\xi}: \mathcal{B}(R) \rightarrow[0,1]$, we can define interval-valued expected value $\widehat{E}(z)$ by the same formula.

Definition 4.3 We say that an IV-observable $z$ is an integrable IV-observable, if the integral $\int_{R} t d k_{z}(t)$ exists. In this case, we define an interval-valued expected value (IV-expected value) $\widehat{E}(z)$ by

$$
\widehat{E}(z)=\int_{R} t d k_{z}(t)
$$

If the integral $\int_{R} t^{2} d k_{z}(t)$ exists, then we define interval-valued dispersion (IV-dispersion) $\widehat{D}^{2}(x)$ by the formula

$$
\widehat{D}^{2}(z)=\int_{R} t^{2} d k_{z}(t)-(\widehat{E}(z))^{2}=\int_{R}(t-\widehat{E}(z))^{2} d k_{z}(t)
$$


A very important notion is the notion of distribution function, as the following definition states.

Definition 4.4 If $z: \mathcal{B}(R) \rightarrow \mathcal{K}$ is an IV-observable, and $k: \mathcal{K} \rightarrow[0,1]$ is an IV-state, then the interval-valued distribution function (IV-distribution function) of $z$ is the function $\widehat{F}: R \rightarrow[0,1]$ defined by the formula

$$
\widehat{F}(t)=k(z((-\infty, t)))
$$

for each $t \in R$.

Of course, the IV-distribution function fulfils the same properties as the classical distribution function. We show a connection between IV-distribution function and IF-distribution function, too. Recall that by intuitionistic fuzzy distribution function (IF-distribution function) of an IF-observable $x: \mathcal{B}(R) \rightarrow \mathcal{F}$ we understand each function $\mathbf{F}: R \rightarrow[0,1]$ defined by the formula

$$
\mathbf{F}(t)=\mathbf{m}(x((-\infty, t)))
$$

for each $t \in R$, where $\mathbf{m}: \mathcal{F} \rightarrow[0,1]$ is an IF-state.

Theorem 4.5 Let $\widehat{F}: R \rightarrow[0,1]$ be the IV-distribution function of an IV-observable $z: \mathcal{B}(R) \rightarrow$ $\mathcal{K}$. Then $\widehat{F}$ is non-decreasing on $R$, left continuous in each point $t \in R$ and

$$
\lim _{t \rightarrow-\infty} \widehat{F}(t)=0, \lim _{t \rightarrow \infty} \widehat{F}(t)=1
$$

Moreover,

$$
\widehat{F}(t)=\mathbf{F}(t),
$$

for each $t \in R$, where $\mathbf{F}$ is an IF-distribution function of an IF-observable $x: \mathcal{B}(R) \rightarrow \mathcal{F}$.

Proof. Let $\widehat{F}: R \rightarrow[0,1]$ be the IV-distribution function of an IV-observable $z: \mathcal{B}(R) \rightarrow \mathcal{K}$. Then by Theorem 4.1 and Definition 4.4 we have

$$
\widehat{F}(t)=k(z((-\infty, t)))=k_{z}((-\infty, t))=\mathbf{m}_{x}((-\infty, t))=\mathbf{m}(x((-\infty, t)))=\mathbf{F}(t),
$$

for each $t \in R$, where $\mathbf{F}(t)$ is an IF-distribution function.

Since $\mathbf{F}$ is an IF-distribution function, then it is non-decreasing on $R$, left continuous in each point $t \in R$ and $\lim _{t \rightarrow-\infty} \mathbf{F}(t)=0, \lim _{t \rightarrow \infty} \mathbf{F}(t)=1$ (see [9]). Hence $\widehat{F}$ is non-decreasing on $R$, left continuous in each point $t \in R$ and

$$
\lim _{t \rightarrow-\infty} \widehat{F}(t)=\lim _{t \rightarrow-\infty} \mathbf{F}(t)=0, \lim _{t \rightarrow \infty} \widehat{F}(t)=\lim _{t \rightarrow \infty} \mathbf{F}(t)=1 .
$$

This completes the proof.

Remark 4.6 Theorem 4.5 says that an IV-observable $z$ and an IF-observable $x$ have the same distribution functions. 
Theorem 4.7 Let $\widehat{F}: R \longrightarrow[0,1]$ be the IV-distribution function of an IV-observable $z: \mathcal{B}(R) \rightarrow \mathcal{K}$. Then,

$$
\begin{aligned}
\widehat{E}(z) & =\int_{R} t d \widehat{F}(t) \\
\widehat{D}^{2}(z) & =\int_{R} t^{2} d \widehat{F}(t)-(\widehat{E}(z))^{2}=\int_{R}(t-\widehat{E}(z))^{2} d \widehat{F}(t)
\end{aligned}
$$

Proof. Since $\widehat{F}$ is the IV-distribution function of the probability distribution $k_{z}$, we have

$$
\lambda_{\widehat{F}}([a, b))=\widehat{F}(b)-\widehat{F}(a)=k_{z}([a, b))
$$

hence,

$$
\lambda_{\widehat{F}}=k_{z}
$$

Therefore,

$$
\int_{R} t d \widehat{F}(t)=\int_{R} t d \lambda_{\widehat{F}}(t)=\int_{R} t d k_{z}(t)=\widehat{E}(z) .
$$

Similarly the other equality can be obtained.

\section{Conclusion}

In this paper, we illustrated the connection between intuitionistic fuzzy observable $x: \mathcal{B}(R) \rightarrow \mathcal{F}$ and interval-valued observable $z: \mathcal{B}(R) \rightarrow \mathcal{K}$ and that is $z=\psi \circ x$. We proved the equality of distribution functions in interval-valued and intuitionistic fuzzy case. Therefore, the equality of mean values and the equality of dispersions result from this in interval-valued and intuitionistic fuzzy case, too.

\section{References}

[1] Atannasov, K. (1999). Intuitionistic Fuzzy Sets: Theory and Applications. Springer Physica Verlag, Heidelberg.

[2] Atanassov, K. T. (2012). On Intuitionistic Fuzzy Sets Theory. Springer, Berlin.

[3] Král, P., \& Riečan, B. (2010). Probabilty on interval-valued Events. Proceeding of Eleventh Int. Workshop on GNs and Second Int. Workshop on GNs, IFSs, KE, London, 9-10 July 2010, 43-47.

[4] Lendelová, K., \& Michalíková, A. (2005). Probability on a Lattice $L^{1}$. Proceedings East West Fuzzy Colloquium 2005, 12th Zittau Fuzzy Colloquium, September 21-23, 2005, Zittau Germany, Heft 84/2005 Nr. 2090-2131, Germany: Institut für Prozeßtechnik, Prozeßautomatisierung und Meßtechnik, 79-83. 
[5] Lendelová, K., \& Riečan, B. (2004). Weak law of large numbers for IF-events. Current Issues in Data and Knowledge Engineering (Bernard De Baets et al. eds.), EXIT, Warszawa, 309-314.

[6] Michalíková, A., \& Riečan, B. (2018). On some methods of study of states on intervalvalued fuzzy sets. Notes on Intuitionistic Fuzzy Sets, 24 (4), 5-12.

[7] Riečan, B. (2006). On a problem of Radko Mesiar: general form of IF-probabilities. Fuzzy Sets and Systems, 152, 1485-1490.

[8] Riečan, B. (2006). On the probability and random variables on IF events. In Applied Artifical Intelligence, Proc. 7th FLINS Conf. Genova (D. Ruan et al. eds.), 138-145.

[9] Riečan, B. (2012). Analysis of fuzzy logic models, Intelligent systems (V. Koleshko ed.), INTECH, 219-244.

[10] Zadeh, L. A. (1975). The concept of linguistic variable and its application to approximate reasoning I. Information Sciences, 8 (3), 199-249. 